\title{
Doppler spectrum consideration on V2V communication for platooning
}

\author{
Koichi Serizawa $^{\text {a)}}$, Kazuma Tomimoto, Masayuki Miyashita, \\ and Ryo Yamaguchi
}

Advanced Technology Division, Softbank Corp.,

Telecom Center Building East Tower 13F,

2-5-10 Aomi, Koto-ku, Tokyo 135-0064, Japan

a)koichi.serizawa01@g.softbank.co.jp

Abstract: 5th generation mobile communication (5G) is being actively researched and developed. We focus on "Ultra-Reliable and Low-Latency Communication" and consider the application of truck platooning as a use case. The latest research on the Doppler spectrum of the vehicle-to-vehicle $(\mathrm{V} 2 \mathrm{~V})$ direct communication of platooning vehicles has revealed that the direct wave is not Doppler-shifted and that a component with twice the maximum Doppler frequency was observed.

This paper focuses on road surface reflections and scattered waves, and shows that there is no Doppler shift near the regular reflection point and that the Doppler shift near the transmission/receiving point.

Keywords: 5G mobile communication system, V2V direct communication, platooning, Doppler spectrum, Doppler shift

Classification: Antennas and Propagation

\section{References}

[1] M. Mikami, R. Yamaguchi, and H. Yoshino, "Field experimental evaluation on 5G low latency communications for application to truck platooning," IEICE Technical Report, RCS2018-210, pp. 181-186, Nov. 2018.

[2] K. Seki and M. Hamaguchi, "A study on inter-vehicle communication for truck platooning (2nd Report)," Proc. 17th ITS World Congress, Busan, South Korea, Oct. 2010.

[3] A. Keiji, "Current activities of development on the automated truck platoon - On development status of automated driving technology —," IPSJ Magazine, vol. 54, no. 4, pp. 303-309, Sept. 2013.

[4] K. Serizawa, K. Tomimoto, M. Miyashita, and R. Yamaguchi, "Doppler spectrum evaluation on $\mathrm{V} 2 \mathrm{~V}$ communication for platooning," IEICE Commun. Express, vol. 8, no. 5, pp. 184-189, May 2019. DOI:10.1587/comex. 2019XBL0008 


\section{Introduction}

The advent of 5th mobile communication $(5 \mathrm{G})$ has triggered intense research and development activities to understand the radio wave propagation characteristics of the new frequency band expected to be used. $5 \mathrm{G}$ has three main features: Enhanced Mobile BroadBand (eMBB), multiple simultaneous connections (mMTC), and high-reliability and low-latency (URLLC). Among them, we focus on URLLC, and considering truck platooning as a one of use case [1].

Truck platoons are controlled by electronic connection technology (direct vehicle-to-vehicle communication), and multiple trucks are packed together to form a train. Japan is investigating whether it is possible to man the lead vehicle with all following vehicles being unmanned. This goal is to significantly reduce labor costs, offset the driver shortage problem, increase operation efficiency, and reduce energy consumption $[2,3]$.

We measure the Doppler shift present in platooning V2V direct communication, and pay close attention to the spectral broadening. In previous letter, when directional antenna was used during platooning, twice the maximum Doppler frequency was observed, and it was described that multiple scattering by the road surface and vehicles was the cause [4]. In this letter, the spectrum spread up to the maximum Doppler frequency is observed, using an omnidirectional antenna. It is experimentally clarified that the cause is the difference between the incident angle/exit angle of the road scattered wave.

\section{Convoy travel V2V Doppler spectrum}

\subsection{Measurement overview}

Fig. 1(a) outlines the experimental apparatus used to assess the Doppler spectrum in platooning $\mathrm{V} 2 \mathrm{~V}$ direct communication. Two trucks were lined up, with the leading vehicle as the receiving system and the following vehicle as the transmitting system. For the transmission system, a standard signal generator (SG) was used to transmit an unmodulated continuous wave (CW signal). A spectrum analyzer (SA) was used as receiving system. In order suppress frequency stability concerns, rubidium oscillators using GNSS signals were installed in the transmission and reception systems to synchronize the reference signal.

Fig. 1(d) shows the measurement parameters. The measurements were made in the $28 \mathrm{GHz}$ band (center frequency $27.9 \mathrm{GHz}$ ) used by $5 \mathrm{G}$. Here, the maximum Doppler frequency $f_{\mathrm{D}}$ is expressed by Eq. (1).

$$
f_{\mathrm{D}}=(v / c) \times f_{\mathrm{c}}
$$

$v$ : travel speed, $c$ : speed of light, $f_{\mathrm{c}}$ : Transmitter center frequency

The maximum Doppler frequency, $f_{\mathrm{D}}$, is $775 \mathrm{~Hz}$ and $1550 \mathrm{~Hz}$, as given by Eq. (1) when the center frequency is $27.9 \mathrm{GHz}$ and the moving speed is $30 \mathrm{~km} / \mathrm{h}$ and $60 \mathrm{~km} / \mathrm{h}$. The distance between vehicles in platooning is expected to be $4 \mathrm{~m}$ to $8 \mathrm{~m}$ so as to reduce air resistance and improve fuel efficiency, but for this measurement the separation was kept to about $10 \mathrm{~m}$ for safety as both vehicles were manned. Patterns were measured for two types of transmitting and receiving antennas: an omnidirectional antenna and a quad-ridge horn antenna. The antenna 
height was $0.75 \mathrm{~m}$ at both the transmitter and receiver. Antennas are generally installed as high as possible, but as most trucks will carry containers owned someone else, the antenna height is determined by the rear chassis arrangement. Using the same antenna height simplifies the measurement setup.

\subsection{Platooning and Doppler spectrum}

Both the leading car and the following car were driven at the same speed, and the Doppler spectrum was observed. Fig. 1(b) and 1(c) show the spectra observed when omnidirectional antennas and quad-ridge horn antennas were used, respectively. The horizontal axis shows the frequency shift (frequency difference from the transmission frequency), and the vertical axis shows the reception level. The spectrum without Doppler shift is considered to be the direct wave and road surface regular reflection waves. From Fig. 1(b), when the omnidirectional antenna is used, the spectrum spreads with a gentle Doppler shift of $1500 \mathrm{~Hz}$ and $3000 \mathrm{~Hz}$, at the
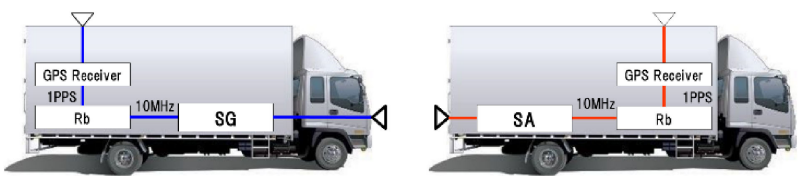

(a): Outline of experimental equipment

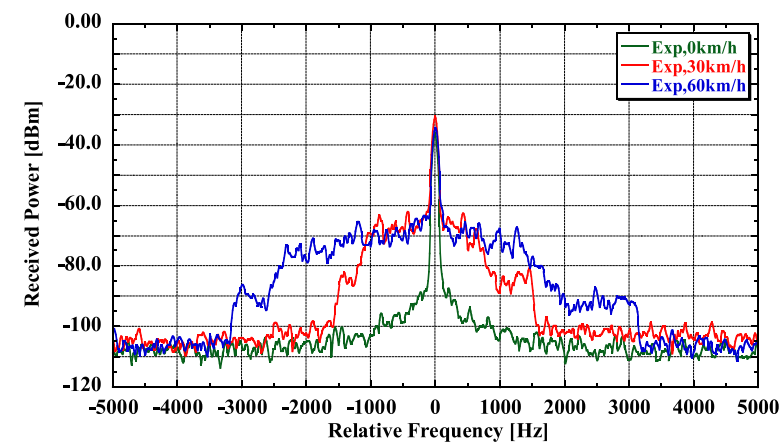

(b): Doppler spectrum measurement results

(When platooning, Omnidirectional antenna)

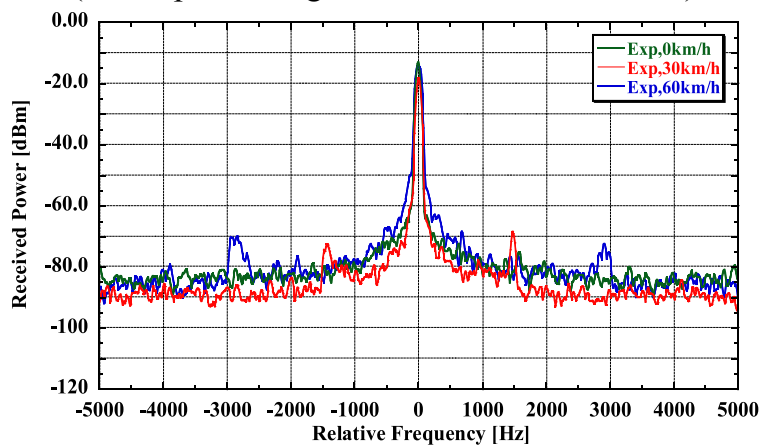

(c): Doppler spectrum measurement results

(When platooning, quad-ridge horn antenna) [4]

(d): Measurement parameters 
driving speeds of $30 \mathrm{~km} / \mathrm{h}$ and $60 \mathrm{~km} / \mathrm{h}$, respectively. In Fig. 1(c), a weak Doppler shifted spectrum was observed at about $1500 \mathrm{~Hz}$ and $3000 \mathrm{~Hz}$ [4]. These spectra are approximately twice the maximum Doppler frequency. These double waves are caused by multiple waves scattered from stationary features such as road surfaces [4]. Here, in order to clarify the cause of the gentle spectrum broadening in Fig. 1(b), we focused on the waves scattered from the road surface.

\section{Experiment on road surface scattered waves}

As a result of measuring the Doppler shift, spectral broadening was observed when the omnidirectional antenna was used. In order to identify the cause, we paid close attention to the incident angle/exit angle of the road surface reflection/scattered wave and confirmed the influence of Doppler shift.

\subsection{Examination of road scattering model}

Fig. 2(a) shows a Doppler shift model for platooning. Given that the following truck transmits while the leading truck receives,

(1) $\mathrm{CW}$ transmission signal with the carrier frequency $f_{\mathrm{c}}$ is transmitted while the following vehicle is running. At this time, as the transmitting antenna is moving forwards the frequency is raised by $\Delta f$.

(1) 'As the receiving antenna is moving forwards at the same speed, the received frequency is lowered by $\Delta f$ so,

$$
f_{\mathrm{Rx}}=f_{\mathrm{c}}+\Delta f-\Delta f=f_{\mathrm{c}} .
$$

(2) The incident wave on the road surface changes frequency according to the angle between the road surface and the antenna, and becomes $f_{\mathrm{Tx}}$ as per Eq. (2).

$$
f_{T x}=f_{\mathrm{c}}+f_{\mathrm{c}} \times(v / c) \times \cos \theta_{\mathrm{Tx}}
$$

(3) When the road surface wave reaches the receiving antenna, the frequency changes according to the angle between the road surface and the antenna, as in Eq. (2), and becomes $f_{\mathrm{Rx}}$ as per Eq. (3). At this time, the frequency is lowered because the receiving antenna is moving away from the reflection point.

$$
\begin{aligned}
f_{R x} & =f_{\mathrm{c}}+f_{\mathrm{c}} \times(v / c) \times \cos \theta_{\mathrm{Tx}}-f_{\mathrm{c}} \times(v / c) \times \cos \theta_{\mathrm{Rx}} \\
& =f_{\mathrm{c}}\left\{1+(v / c)\left(\cos \theta_{\mathrm{Tx}}-\cos \theta_{\mathrm{Rx}}\right)\right\}
\end{aligned}
$$

The above formula shows that with normal reflection, $\theta_{\mathrm{Tx}}=\theta_{\mathrm{Rx}}$, there is no Doppler shift. Moreover, for the $\left(\theta_{\mathrm{Tx}}, \theta_{\mathrm{Rx}}\right)$ values of $\left(90^{\circ}, 0^{\circ}\right),\left(0^{\circ}, 90^{\circ}\right)$, the Doppler shift at the time of reception is roughly $-f_{\mathrm{D}}, f_{\mathrm{D}}$. As shown in Fig. 2(a), at each road surface scattering point, $\theta_{\mathrm{Tx}}, \theta_{\mathrm{Rx}}$ is uniquely determined, $-1<$ $\left(\cos \theta_{\mathrm{TX}}-\cos \theta_{\mathrm{Rx}}\right)<1$, the Doppler frequency received by the first car, $f_{R x}$, lies in the range $-f_{\mathrm{D}}<f_{R x}<f_{\mathrm{D}}$. Fig. 2(b) shows that for $10 \mathrm{~m}$ of Inter-vehicular distance, the distance from the point immediately below the transmitting antenna to the road surface scattering point and the normalized Doppler shift frequency related by $\left\{f_{\mathrm{D}} \times\left(\cos \theta_{\mathrm{Tx}}-\cos \theta_{\mathrm{Rx}}\right)\right\}$. From this figure, it can be seen that the road surface scattered wave doppler shifts by $-f_{\mathrm{D}}$ near the transmission point and doesn't doppler shift over a wide range near the normal reflection point as the intermediate point. On the other hand, it can be confirmed that $\mathrm{a}+f_{\mathrm{D}}$ shift occurs in near the 
receiving point. This is a characteristic corresponding to the difference between the transmission angle $\theta_{\mathrm{TX}}$ and the reception angle $\theta_{\mathrm{RX}}$ as described above.

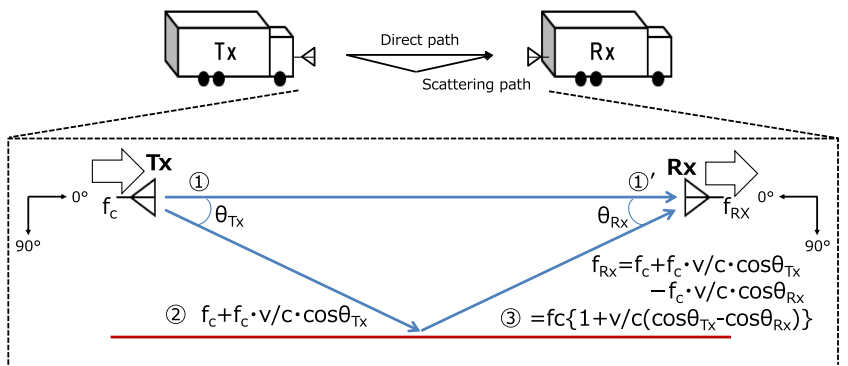

(a): Study of Doppler shift due to road surface scattering path

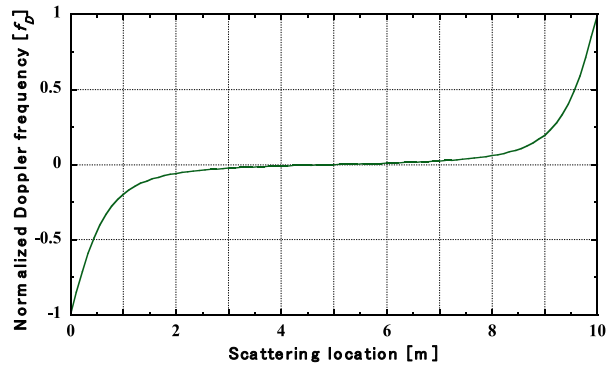

(b): Normalized Doppler shift vs. location of road surface scattering points

Fig. 2. Examinations of scattering path

\subsection{Verification of road surface scattering model}

For the propagation paths from the transmitting antenna to the receiving antenna of $\left(90^{\circ}, 5.5^{\circ}\right)$ and $\left(0^{\circ}, 5.5^{\circ}\right)\left(\theta_{\mathrm{Tx}}, \theta_{\mathrm{Rx}}\right.$, respectively), the Doppler frequencies at the time of reception were assumed to be approximately $-f_{\mathrm{D}}$ and $f_{\mathrm{D}}$, respectively. This verification experiment used quad-ridge horn antennas used as the transmitting and receiving antennas, and the (transmitting antenna angle and receiving antenna angle) were set to $\left(90^{\circ}, 0^{\circ}\right),\left(0^{\circ}, 90^{\circ}\right)$ to limit the road surface scattering path; measurements were conducted to determine if a Doppler shifted spectrum could be observed in the vicinity of $-f_{\mathrm{D}}$ and $f_{\mathrm{D}}$. Fig. 3(a) shows the measurement parameters for the verification experiment, while Fig. 3(b) and (c) show the measurement results. Fig. 3(b) shows that the received spectrum level near $-f_{\mathrm{D}}$ is high because the transmitting antenna is tilted downwards at $90^{\circ}$. On the other hand, in Fig. 3(c), the level of the received spectrum near $f_{\mathrm{D}}$ is high. Therefore, the scattered wave from points, especially near the transmission and receiving point, away from the median reflection point on the road surface are considered to be Doppler shifted.

(a): Measurement parameters

\begin{tabular}{|c|c|}
\hline Center frequency & $\mathbf{2 7 . 9} \mathbf{~ G H z}$ \\
\hline Frequency span & $\mathbf{1 0 ~} \mathbf{~ H z}$ \\
\hline Transmitted signal & $\mathbf{C W}$ \\
\hline Transmission output & $\mathbf{1 5} \mathbf{~ d B m}$ \\
\hline RBW & $\mathbf{5 0 ~ H z}$ \\
\hline VBW & $\mathbf{5 0 ~ H z}$ \\
\hline Sweep time & $\mathbf{1 0 0 0} \mathbf{~ m s}$ \\
\hline Inter-vehicular distance & $\mathbf{1 0 ~} \mathbf{~}$ \\
\hline Antenna & Quad-ridge horn \\
\hline Antenna height & $\mathbf{0 . 7 5}$ m \\
\hline Polarization plane & V polarization \\
\hline
\end{tabular}




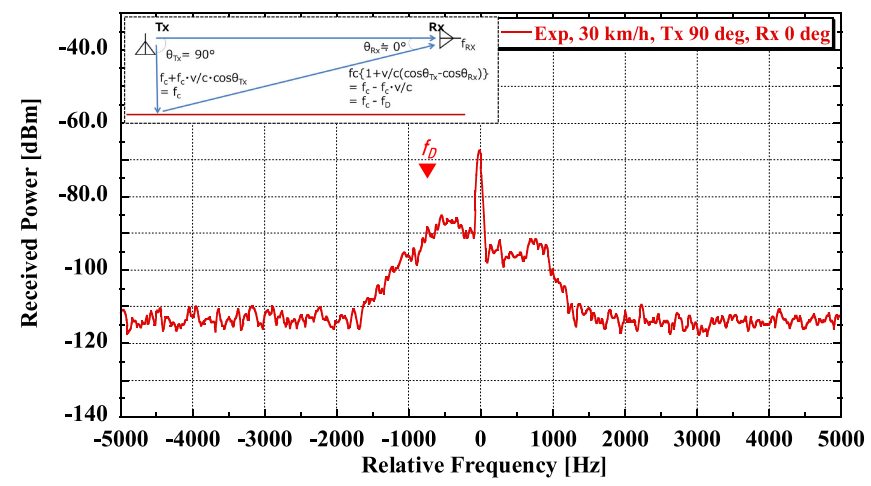

(b): Received power distribution $\left(\theta_{\mathrm{Tx}}=90^{\circ}, \theta_{\mathrm{Rx}}=0^{\circ}\right)$

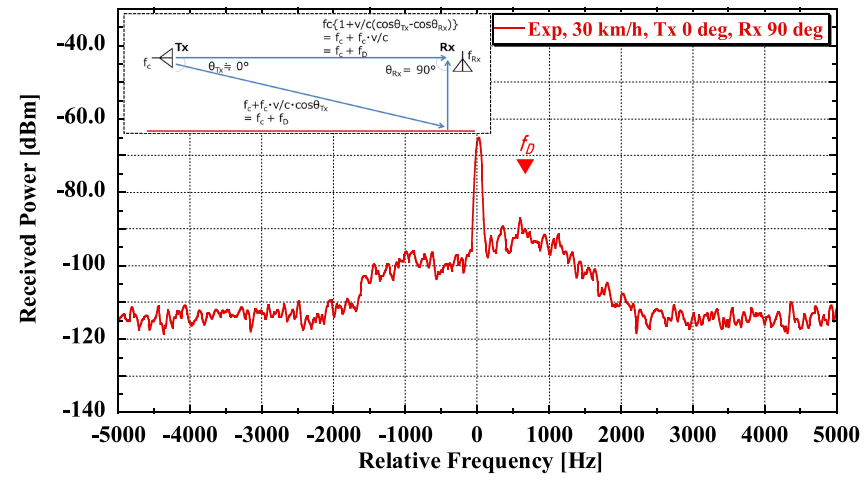

(c): Received power distribution $\left(\theta_{\mathrm{Tx}}=0^{\circ}, \theta_{\mathrm{Rx}}=90^{\circ}\right)$

Fig. 3. Verification setup and measurement results

From these results, the gradual spread of the spectrum during platooning when using omnidirectional antennas is caused by the road surface incident angle/exit angle, shifting from the median reflection point, which yields the Doppler shift found.

\section{Conclusion}

We measured the Doppler spectrum for V2V direct communication using two platooned trucks; the spectrum spreading caused by waves reflected from road surfaces points was examined. Measurements showed that the regular reflected wave (reflection point is equidistant from $\mathrm{Rx}$ and $\mathrm{Tx}$ ) exhibits no spectrum spreading. Furthermore, it was shown that non-regular scattered waves exhibit a broadened spectrum due to the difference between the road incident angle and the exit angle. It was quantitatively shown that road reflection points closer to the Tx antenna lower the Doppler shift, and reflection points closer to the Rx antenna raise the Doppler shift. In the future, we plan to conduct more detailed verification tests in environments closer to real-world use cases, such as when a truck train is overtaken.

\section{Acknowledgments}

This work was partially sponsored by the Ministry of Internal Affairs and Communications of Japan, under the grant, "Research and examination on technical requirements and others in $5 \mathrm{G}$ mobile communication system for realizing low latency communication of over-the-air $1 \mathrm{~ms}$ and end-to-end $10 \mathrm{ms"}$. 